\title{
RANDOM POLYNOMIALS OF HIGH DEGREE AND LEVY CONCENTRATION OF MEASURE *
}

\author{
BERNARD SHIFFMAN ${ }^{\dagger}$ AND STEVE ZELDITCH ${ }^{\dagger}$
}

To Yum-Tong Siu on his 60th birthday

\begin{abstract}
We show that the $\mathcal{L}^{p}$ norms of random sequences of holomorphic sections $s_{N} \in$ $H^{0}\left(M, L^{N}\right)$ of powers of a positive line bundle $L$ over a compact Kähler manifold $M$ satisfy

$$
\left\|s_{N}\right\|_{p} /\left\|s_{N}\right\|_{2}=\left\{\begin{array}{ll}
O(1) & \text { for } 2 \leq p<\infty \\
O(\sqrt{\log N}) & \text { for } p=\infty
\end{array}\right\} \text { almost surely. }
$$

This estimate also holds for almost-holomorphic sections of positive line bundles on symplectic manifolds (in the sense of our previous work) and we give almost sure bounds for the $\mathcal{C}^{k}$ norms. Our methods involve asymptotics of Bergman-Szegö kernels and the concentration of measure phenomenon.
\end{abstract}

1. Introduction. Levy concentration of measure occurs when Lipschitz continuous functions $f$ on a metric probability space $(X, d, \mu)$ of large dimension $d$ are highly concentrated around their median values $\mathcal{M}_{f}$. In the fundamental case where $X$ is the unit $N$-sphere $S^{N}$ with the usual distance function, and $\mu$ is the $\mathrm{SO}(N+1)$-invariant probability measure, the concentration of measure inequality says that

$$
\operatorname{Prob}\left\{x \in S^{N}:\left|f(x)-\mathcal{M}_{f}\right| \geq r\right\} \leq \exp \left(-\frac{(N-1) r^{2}}{2\|f\|_{L i p}^{2}}\right)
$$

where

$$
\|f\|_{L i p}=\sup _{d(x, y)>0} \frac{|f(x)-f(y)|}{d(x, y)}
$$

is the Lipschitz norm. (See, e.g. [Le].)

The purpose of this paper is to apply the concentration of measure inequality for high-dimensional spherical and (associated) Gaussian ensembles to determine the distribution of $\mathcal{L}^{p}$ norms of random complex polynomials and more general holomorphic sections of positive line bundles over complex manifolds. In each case, we have a sequence of (finite dimensional) Hilbert spaces $\mathcal{H}_{N}$ of dimensions $d_{N} \rightarrow \infty$. We will use a natural inner product $\langle,\rangle_{N}$ on $\mathcal{H}_{N}$ to define the unit sphere $S \mathcal{H}_{N} \subset \mathcal{H}_{N}$ endowed with Haar probability measure $\nu_{N}$. We also consider the closely related Gaussian measure $\gamma_{N}$ on $\left(\mathcal{H}_{N},\langle,\rangle_{N}\right)$. In our applications, $\mathcal{H}_{N}$ will belong to one of the following classes:

\section{Complex Ensembles}

i) The space $\mathcal{P}_{\mathbb{C} N}^{m}$ of complex holomorphic polynomials of degree $N$, with the usual Fubini-Study inner product;

ii) More generally, the spaces $H^{0}\left(M, L^{N}\right)$ of holomorphic sections of high powers of a positive hermitian line bundle $L$, with the inner product induced by the Hermitian metric;

\footnotetext{
*Received March 26, 2003; accepted for publication September 5, 2003.

${ }^{\dagger}$ Department of Mathematics, Johns Hopkins University, Baltimore, MD 21218, USA (shiffman@math.jhu.edu, zelditch@math.jhu.edu). Research partially supported by NSF grants DMS0100474 (first author) and DMS-0071358 (second author).
} 
iii) Even more generally, the spaces $H_{J}^{0}\left(M, L^{N}\right)$ of almost-holomorphic sections of an ample line bundle $L \rightarrow M$ over a symplectic almost complex manifold.

The generalization to almost complex symplectic manifolds is motivated by the role that almost holomorphic sections have played in symplectic geometry since the paper of Donaldson [Do]. Almost holomorphic sections behave less 'deterministically' than holomorphic sections do, e.g. their zeros sets may or may not be symplectic submanifolds. This suggests studying them probabilistically. In [ShZe2] we developed the analytic tools sufficient for this purpose, and initiated a probabilistic study in [ShZe3]. The results of this paper on the almost complex case are relevant to, and give a natural continuation of, these earlier articles.

With certain modifications, our methods also apply to:

\section{Real Ensembles}

i) The space $V_{N}\left(S^{m}\right)$ of spherical harmonics of degree $N$, with the standard inner product;

ii) The space $\mathcal{P}_{\mathbb{R} N}^{m}$ of real polynomials of degree $N$, with the 'Fock space' inner product;

iii) The space $\mathcal{E}_{\lambda, M, g}$ of linear combinations of eigenfunctions of the Laplacian on a compact Riemannian manifold $(M, g)$ with eigenvalue of $\sqrt{\Delta}$ in the interval $[\lambda, \lambda+1]$.

The pattern of results and techniques is similar in the real and complex cases. The main difference is in the properties of their reproducing (Szegö) kernels. The theory of random real ensembles is not as developed as the complex case, and we plan to devote a separate article for that purpose. Some of the relevant steps have already been taken in [Ne, Va, Ze1].

The main functionals we consider are the norms on $S \mathcal{H}_{N}$ :

$$
\mathcal{L}^{p}(s)=\|s\|_{p} \quad(2 \leq p \leq \infty), \quad \mathcal{L}_{(k)}^{\infty}(s)=\left\|\nabla^{k} s\right\|_{\infty} \quad(k=1,2,3, \ldots) .
$$

We separate out the cases $p=\infty, p<\infty$ since the proofs are somewhat different. We also separate out the cases $k=0, k>0$ since the case $k=0$ is more elementary.

Our first result gives concentration inequalities for sup norms. The same results hold for both real and complex ensembles, but we only carry out the proof in the complex case. We write elements of $\mathcal{H}_{N}$ as $s_{N}$.

THEOREM 1.1. For each of the above complex ensembles, there exist constants $C>0$ such that:

$$
\nu_{N}\left\{s_{N} \in S \mathcal{H}_{N}: \sup _{M}\left|s_{N}\right|>C \sqrt{\log N}\right\}<O\left(\frac{1}{N^{2}}\right)
$$

In fact, for any $k>0$, we can bound the probabilities by $O\left(N^{-k}\right)$ by choosing $C$ to be sufficiently large.

As a corollary we obtain almost sure bounds on the growth of $\mathcal{L}^{\infty}$ norms for independent random sequences of $\mathcal{L}^{2}$-normalized holomorphic sections. To state the result, we introduce the probability sequence space $\mathcal{S}=\prod_{N=1}^{\infty} S \mathcal{H}_{N}$ with the measure $\nu=\prod_{N=1}^{\infty} \nu_{N}$. The estimate of Theorem 1.1 immediately implies that

$$
\limsup _{N \rightarrow \infty} \frac{\sup _{X}\left|s_{N}\right|}{\sqrt{\log N}} \leq C \quad \text { almost surely . }
$$


Hence we have:

COROLlary 1.2. Sequences of sections $s_{N} \in S \mathcal{H}_{N}$ satisfy:

$$
\left\|s_{N}\right\|_{\infty}=O(\sqrt{\log N}) \quad \text { almost surely. }
$$

Results of this type on sup norms were first proved by Salem-Zygmund [SaZy] in the case of random trigonometric polynomials on the circle, and by Kahane [Ka] for random trigonometric polynomials on tori. Vanderkam [Va] generalized the results to the case of random spherical harmonics by a geometric method that seems special to the sphere. Nonnenmacher-Voros $[\mathrm{NV}]$ obtained bounds on sup norms for random theta functions on elliptic curves using properties of the Szegö kernel in that setting. Neuheisel $[\mathrm{Ne}]$ adapted their method to simplify the sup norm estimates of [Va] on random spherical harmonics. The contribution of this paper is to give simple and general results by using properties of Szegö kernels and methods related to Levy concentration of measure.

Our second result gives $\mathcal{L}^{p}$ bounds on such sequences.

TheOREM 1.3. Let $\operatorname{dim} M=m$ and let $2 \leq p<\infty$. Then for each complex ensemble, the median values of the $\mathcal{L}^{p}$ norm on the unit spheres $S \mathcal{H}_{N}$ are bounded by a constant $\alpha=\alpha(p, m)$, and

$$
\nu_{N}\left\{s_{N} \in S \mathcal{H}_{N}: \mathcal{L}^{p}\left(s_{N}\right)>r+\alpha\right\} \leq \exp \left(-C r^{2} N^{2 m / p}\right)
$$

for some constant $C>0$. Hence, sequences of sections $s_{N} \in S \mathcal{H}_{N}$ satisfy $\left\|s_{N}\right\|_{p}=$ $O(1)$ almost surely.

Our final results pertain to $\mathcal{C}^{k}$ norms. Almost sure estimates on $\mathcal{C}^{k}$ norms must take into account the off-diagonal behavior of the reproducing kernels as well as the on-diagonal behavior. One of our motivations here is to show that sequences of increasing degree of almost holomorphic sections of ample line bundles over symplectic manifolds almost always have properties similar to the asymptotically holomorphic sections of Donaldson [Do]. One of these latter properties is a nearly bounded $\mathcal{L}^{\infty}$ norm. The following result shows that almost surely, the sup norms of $\mathcal{L}^{2}$-normalized almost holomorphic sections satisfy comparable estimates to asymptotically holomorphic sections.

THEOREM 1.4. Let $\operatorname{dim} M=m$ and let $j \in \mathbb{Z}^{+}$. Then there is a positive constant $C=C_{m j}$ such that for each complex ensemble,

$$
\nu_{N}\left\{s_{N} \in S \mathcal{H}_{N}: \sup _{M}\left|\nabla^{k} s_{N}\right|>C \sqrt{N^{k} \log N}\right\} \leq O\left(N^{-j}\right), \quad \text { for } k=1,2,3, \ldots
$$

Thus sequences $s_{N} \in S \mathcal{H}_{N}$ satisfy

- $\left\|\nabla^{k} s_{N}\right\|_{\infty}=O\left(\sqrt{N^{k} \log N}\right)$ almost surely.

If $(M, L, \omega)$ is symplectic, then sequences $s_{N} \in S H_{J}^{0}\left(M, L^{N}\right)$ of almost-holomorphic sections additionally satisfy:

- $\left\|\bar{\partial} s_{N}\right\|_{\infty}=O(\sqrt{\log N})$ almost surely.

- $\left\|\nabla^{k} \bar{\partial} s_{N}\right\|_{\infty}=O\left(\sqrt{N^{k} \log N}\right)$ almost surely.

We can also endow $\left(\mathcal{H}_{N},\langle,\rangle_{N}\right)$ with the Gaussian probability measure $\gamma_{N}$ given by:

$$
d \gamma_{N}(s)=\frac{1}{\pi^{d_{N}}} \exp \left(-\sum_{j=1}^{d}\left|c_{j}\right|^{2}\right) d c, \quad s=\sum_{j=1}^{d_{N}} c_{j} S_{j}^{N}
$$


where $\left\{S_{j}^{N}\right\}$ is an orthonormal basis of $\mathcal{H}_{N}$. Thus, the coefficients $c_{j}$ are independent complex Gaussian random variables satisfying:

$$
\mathbf{E}\left(c_{j}\right)=0, \quad \mathbf{E}\left(c_{j} c_{k}\right)=0, \quad \mathbf{E}\left(c_{j} \bar{c}_{k}\right)=\delta_{j k}
$$

where $\mathbf{E}$ denotes the expected value. It follows immediately from our above results that for sequences $\left\{s_{N}\right\} \in \prod_{N=1}^{\infty} \mathcal{H}_{N}$, with probability measure $\gamma=\prod_{N=1}^{\infty} \gamma_{N}$, we have:

- $\left\|s_{N}\right\|_{\infty} /\left\|s_{N}\right\|_{2}=O(\sqrt{\log N})$ almost surely.

- $\left\|s_{N}\right\|_{p} /\left\|s_{N}\right\|_{2}=O(1)$ almost surely, for $2 \leq p<\infty$.

- $\left\|\nabla^{k} s_{N}\right\|_{\infty} /\left\|s_{N}\right\|_{2}=O\left(\sqrt{N^{k} \log N}\right)$ almost surely.

- $\left\|\bar{\partial} s_{N}\right\|_{\infty} /\left\|s_{N}\right\|_{2}=O(\sqrt{\log N})$ almost surely.

- $\left\|\nabla^{k} \bar{\partial} s_{N}\right\|_{\infty} /\left\|s_{N}\right\|_{2}=O\left(\sqrt{N^{k} \log N}\right)$ almost surely.

(The last two statements are vacuous in the holomorphic case.)

We close the introduction with some open problems that seem of interest in this area. First, we have assumed throughout that the spaces of polynomials and sections are equipped with Gaussian measures. It would be interesting to know how the results would change if one used other measures, e.g. measures of the form $e^{-S(f)} \mathcal{D} f$ where $S(f)=\|\nabla f\|_{\mathcal{L}^{2}}^{2}+\|f\|_{\mathcal{L}^{2}}^{2}+\beta\|f\|_{\mathcal{L}^{2}}^{4}$ that arise in quantum field theory. These are difficult to analyze as $N \rightarrow \infty$ since little is known about the minimal value of $S$ or the number of its critical points. In the case of $S(f)=\|f\|_{\mathcal{L}^{\infty}}$, it is known [Bo1, Bo2] that inf $S(f)$ as $f$ varies over $S H^{0}\left(\mathbb{C P}^{1}, \mathcal{O}(N)\right)$ is bounded as $N \rightarrow \infty$. Bourgain constructed explicit sequences of $L^{2}$-normalized polynomials of bounded sup-norm using Rudin-Shapiro sequences and an estimate on the $\mathcal{L}^{p}$ mapping norm of the Szegö kernel. We show in Lemma 4.1 that the latter estimate holds for any line bundle over any Kähler manifold.

2. Notation and background. The study of sup-norms of random polynomials has a long history. Among the earliest articles is that of Paley-Wiener-Zygmund [PWZ]. In [SaZy], Salem-Zygmund studied sup-norms of random trigonometrical polynomials

$$
P(t)=\sum_{0}^{N} \epsilon_{n} a_{n} \cos \left(n t+\varphi_{n}\right)
$$

where the $\varepsilon_{n}$ are independent random variables taking the values \pm 1 with probability $\frac{1}{2}$. Their main result is a prototype for the subsequent results:

$$
\operatorname{Prob}\left\{\|P\|_{\infty}<\lambda\left(\sum_{n=1}^{N} a_{n}^{2} \log N\right)^{\frac{1}{2}}\right\} \rightarrow 1, \quad N \rightarrow \infty .
$$

Such estimates are further developed in the book of Kahane [Ka], where further references may be found. In particular, Kahane ([Ka], Section 6.2, Theorem 3) proves a many-variable generalization of Salem-Zygmund's theorem that is a model for our results: Let

$$
P\left(t_{1}, \ldots, t_{m}\right)=\sum_{n=1}^{k} \xi_{n} f_{n}\left(t_{1}, \ldots, t_{m}\right)
$$

where $\left\{f_{n}\right\}$ are complex trigonometric polynomials in $m$ variables of degrees $\leq N$, and where $\xi_{n}$ are normal random variables. Then:

$$
\operatorname{Prob}\left\{\|P\|_{\infty} \geq C\left(s \sum_{n=1}^{k}\left\|f_{n}\right\|_{\infty}^{2} \log N\right)^{\frac{1}{2}}\right\} \leq N^{-2} e^{-s}
$$


Our estimates on sup norms follow however a different path, and involve scaling asymptotics of Bergman-Szegö kernels and concentration of measure estimates, applied to the spaces $\mathcal{H}_{N}$. To describe these Bergman-Szegö kernels, we regard the spaces $\mathcal{H}_{N}$ as subspaces of the Hilbert space $\mathcal{L}^{2}(X)$ of complex-valued squareintegrable functions on a manifold $X\left(=\right.$ the $S^{1}$ bundle associated to the line bundle $L)$. We denote by

$$
\Pi_{N}: \mathcal{L}^{2}(X) \rightarrow \mathcal{H}_{N}
$$

the orthogonal projection onto the subspace; it is given by the Bergman-Szegö kernel

$$
\Pi_{N}(x, y)=\sum_{j=1}^{d_{N}} S_{j}^{N}(x) \overline{S_{j}^{N}(y)}, \quad d_{N}=\operatorname{dim} \mathcal{H}_{N}
$$

where $\left\{S_{j}^{N}\right\}$ is an orthonormal basis of $\mathcal{H}_{N}$ (see $\S 2.2$ ). ments:

The following well-known elementary probability lemma is central to our argu-

LEMma 2.1. Let $A \in S^{2 d-1} \subset \mathbb{C}^{d}$, and give $S^{2 d-1}$ Haar probability measure. Then

$$
\operatorname{Prob}\left\{P \in S^{2 d-1}:|\langle P, A\rangle|>\lambda\right\} \leq e^{-(d-1) \lambda^{2}}
$$

Proof. We can assume without loss of generality that $A=(1,0, \ldots, 0)$. Let

$$
V_{\lambda}=\operatorname{Vol}\left(\left\{P \in S^{2 d-1}:\left|P_{1}\right|>\lambda\right\}\right) \quad(0 \leq \lambda<1),
$$

where Vol denotes $(2 d-1)$-dimensional Euclidean volume. Our desired probability equals $V_{\lambda} / V_{0}$. Let $\sigma_{n}=\operatorname{Vol}\left(S^{2 n-1}\right)=\frac{2 \pi^{n}}{(n-1) !}$. We compute

$$
\begin{aligned}
V_{\lambda} & =\int_{\lambda}^{1} \sigma_{d-1}\left(1-r^{2}\right)^{\frac{2 d-3}{2}} \frac{2 \pi r d r}{\sqrt{1-r^{2}}}=2 \pi \sigma_{d-1} \int_{\lambda}^{1}\left(1-r^{2}\right)^{d-2} r d r \\
& =\frac{\pi \sigma_{d-1}}{d-1}\left(1-\lambda^{2}\right)^{d-1}=\sigma_{d}\left(1-\lambda^{2}\right)^{d-1} .
\end{aligned}
$$

Therefore,

$$
\operatorname{Prob}\left\{P \in S^{2 d-1}:|\langle P, A\rangle|>\lambda\right\}=V_{\lambda} / V_{0}=\left(1-\lambda^{2}\right)^{d-1} \leq e^{-(d-1) \lambda^{2}} .
$$

REMARK. In the real case, from the Levy concentration of measure inequality (1) with $f(X)=\Re\langle P, A\rangle$, we obtain the analogous result:

$$
\operatorname{Prob}\left\{P \in S^{2 d-1}:|\Re\langle P, A\rangle|>\lambda\right\} \leq e^{-(d-1) \lambda^{2}} .
$$

In the complex case, we then have

$$
\begin{aligned}
\operatorname{Prob} & \left\{P \in S^{2 d-1}:|\langle P, A\rangle|>\lambda\right\} \\
& \leq \operatorname{Prob}\left\{P \in S^{2 d-1}:|\Re\langle P, A\rangle|>\lambda / \sqrt{2}\right\}+\operatorname{Prob}\left\{P \in S^{2 d-1}:|\Im\langle P, A\rangle|>\lambda / \sqrt{2}\right\} \\
& \leq 2 e^{-(d-1) \lambda^{2} / 2},
\end{aligned}
$$

which is not as sharp as Lemma 2.1. 
2.1. Complex ensembles. Let us now specify the set-up on Kähler manifolds. We only give a brief discussion and refer the reader to [BSZ1, BSZ2, ShZe1, ShZe2, Ze2] for further details.

The simplest example is where $\mathcal{H}_{N}=\mathcal{P}_{\mathbb{C}}^{N}$, the space of holomorphic polynomials

$$
f\left(z_{1}, \ldots, z_{m}\right)=\sum_{\alpha \in \mathbf{N}^{m}:|\alpha| \leq N} c_{\alpha} z^{\alpha}
$$

Such polynomials may be regarded as sections $s \in H^{0}\left(\mathbb{C} \mathbb{P}^{m}, \mathcal{O}(N)\right)$ of the $N$-th power of the hyperplane section bundle $\mathcal{O}(1) \rightarrow \mathbb{C P}^{m}$. The identification is established by homogenizing each monomial

$$
z_{1}^{\alpha_{1}} \cdots z_{m}^{\alpha_{m}} \rightarrow z_{0}^{N-|\alpha|} z_{1}^{\alpha_{1}} \cdots z_{m}^{\alpha_{m}}
$$

to be homogeneous of degree $N$. The homogenization $\hat{f}$ of $f$ is then determined by its restriction to $S^{2 m+1} \subset \mathbb{C}^{m}$ where it is equivariant under the natural $S^{1}$ action of $S^{2 m+1} \rightarrow \mathbb{C P}^{m}: \hat{f}\left(e^{i \theta} z\right)=e^{i N \theta} \hat{f}(z)$. The space of such homogenized polynomials will be denoted by $\mathcal{H}_{N}$. They satisfy the boundary Cauchy-Riemann equations $\bar{\partial}_{b} \hat{f}=0$ as boundary values of holomorphic functions on the unit ball $B_{1} \subset \mathbb{C}^{m}$.

Essentially the same construction exists on any compact algebraic manifold $(M, \omega)$, i.e. a Kähler manifold such that $\left[\frac{1}{\pi} \omega\right]$ is an integral cohomology class. There exists a hermitian line bundle $(L, h) \rightarrow M$ and a metric connection $\nabla$ on $L$ with curvature given by $\frac{i}{2} \Theta_{L}=\omega$. We denote the space of holomorphic sections of the $N$-th power of $L$ by $H^{0}\left(M, L^{N}\right)$.

In order to simultaneously analyze sections $s \in H^{0}\left(M, L^{N}\right)$ for all $N$, we lift them to the associated $S^{1}$ bundle

$$
X=\left\{v \in L^{*}:\|v\|_{h^{*}}=1\right\} \rightarrow M
$$

where $\pi: L^{*} \rightarrow M$ denotes the dual line bundle to $L$ with dual metric $h^{*}$. We let $\alpha$ be the connection 1 -form on $X$ given by $\nabla$; we then have $d \alpha=\pi^{*} \omega$, and thus $\alpha$ is a contact form on $X$, i.e., $\alpha \wedge(d \alpha)^{m}$ is a volume form on $X$.

We let $r_{\theta} x=e^{i \theta} x(x \in X)$ denote the $S^{1}$ action on $X$ and denote its infinitesimal generator by $\frac{\partial}{\partial \theta}$. A section $s$ of $L$ determines an equivariant function $\hat{s}$ on $L^{*}$ by the rule $\hat{s}(\lambda)=(\lambda, s(z))\left(\lambda \in L_{z}^{*}, z \in M\right)$. We restrict $\hat{s}$ to $X$ to an equivariant function transforming by $\hat{s}\left(r_{\theta} x\right)=e^{i \theta} \hat{s}(x)$. Similarly, a section $s_{N}$ of $L^{N}$ determines an equivariant function $\hat{s}_{N}$ on $X$ : put

$$
\hat{s}_{N}(\lambda)=\left(\lambda^{\otimes N}, s_{N}(z)\right), \quad \lambda \in X_{z}
$$

where $\lambda^{\otimes N}=\lambda \otimes \cdots \otimes \lambda$; then $\hat{s}_{N}\left(r_{\theta} x\right)=e^{i N \theta} \hat{s}_{N}(x)$. We denote by $\mathcal{L}_{N}^{2}(X)$ the space of such equivariant functions transforming by the $N$-th character, and by $\mathcal{H}_{N}$ the subspace of CR functions annihilated by the tangential Cauchy-Riemann operator $\bar{\partial}_{b}$.

The space $\mathcal{H}_{N}$ carries the natural inner product

$$
\langle\hat{s}, \overline{\hat{t}}\rangle=\int_{X} \hat{s} \hat{\hat{t}} d V, \quad d V=\alpha \wedge(d \alpha)^{m-1}
$$

We choose an orthonormal basis $\left\{S_{j}^{N}\right\}$ and write every element as

$$
\hat{s}=\sum_{j=1}^{d_{N}} a_{j} S_{j}^{N} .
$$


By the Riemann-Roch formula, we have the estimate for the dimensions $d_{N}$ :

$$
d_{N}=\frac{c_{1}(L)^{m}}{m !} N^{m}+\mathcal{O}\left(N^{m-1}\right) \text {. }
$$

2.1.1. Almost complex ensembles. This is similar to the complex case except that the complex structure is non-integrable. We let $(M, \omega, J)$ be a compact, almost complex symplectic manifold such that $\left[\frac{1}{\pi} \omega\right]$ is an integral cohomology class, and choose a hermitian line bundle $(L, h) \rightarrow M$ and a metric connection $\nabla$ on $L$ with $\frac{i}{2} \Theta_{L}=\omega$.

In the general almost-complex symplectic case it is an almost CR manifold. The almost $C R$ structure is defined as follows: The kernel of $\alpha$ defines a horizontal hyperplane bundle $H \subset T X$. Using the projection $\pi: X \rightarrow M$, we may lift the splitting $T M=T^{1,0} M \oplus T^{0,1} M$ to a splitting $H=H^{1,0} \oplus H^{0,1}$. The almost CR structure on $X$ is defined to be the splitting $T X=H^{1,0} \oplus H^{0,1} \oplus \mathbb{C} \frac{\partial}{\partial \theta}$. We also consider a local orthonormal frame $Z_{1}, \ldots, Z_{n}$ of $H^{1,0}$, resp. $\bar{Z}_{1}, \ldots, Z_{m}$ of $H^{0,1}$, and dual orthonormal coframes $\vartheta_{1}, \ldots, \vartheta_{m}$, resp. $\bar{\vartheta}_{1}, \ldots, \bar{\vartheta}_{m}$. On the manifold $X$ we have $d=\partial_{b}+\bar{\partial}_{b}+\frac{\partial}{\partial \theta} \otimes \alpha$, where $\partial_{b}=\sum_{j=1}^{m} \vartheta_{j} \otimes Z_{j}$ and $\bar{\partial}_{b}=\sum_{j=1}^{m} \bar{\vartheta}_{j} \otimes \bar{Z}_{j}$. We define the almost-CR $\bar{\partial}_{b}$ operator by $\bar{\partial}_{b}=\left.d f\right|_{H^{1,0}}$. Note that for an $\mathcal{L}^{2}$ section $s_{N}$ of $L^{N}$, we have

$$
\left(\nabla_{L^{N}} s_{N}\right) \widehat{=} d^{h} \hat{s}_{N}
$$

where $d^{h}=\partial_{b}+\bar{\partial}_{b}$ is the horizontal derivative on $X$.

As discussed in [BG, ShZe2], there exists a pseudodifferential perturbation of $\bar{\partial}_{b}$ which has the main properties of $\bar{\partial}_{b}$ in the integrable complex case. We denote its kernel by $\mathcal{H}_{N}$ and refer to [BG, ShZe2] for the definition. By the Riemann-Roch formula of Boutet de Monvel - Guillemin [BG, §14], its dimension $d_{N}$ is again the one in the complex case:

$$
d_{N}=\frac{c_{1}(L)^{m}}{m !} N^{m}+O\left(N^{m-1}\right)
$$

2.2. Bergman-Szegö kernels. We let $\Pi_{N}: \mathcal{L}^{2}(X) \rightarrow \mathcal{H}_{N}(X)$ denote the orthogonal projection. The Bergman-Szegö kernel $\Pi_{N}(x, y)$ is characterized by

$$
\Pi_{N} F(x)=\int_{X} \Pi_{N}(x, y) F(y) d V_{X}(y), \quad F \in \mathcal{L}^{2}(X) .
$$

It can be given as

$$
\Pi_{N}(x, y)=\sum_{j=1}^{d_{N}} S_{j}^{N}(x) \overline{S_{j}^{N}(y)}
$$

where $S_{1}^{N}, \ldots, S_{d_{N}}^{N}$ form an orthonormal basis of $\mathcal{H}_{N}^{2}(X)$.

The Bergman-Szegö kernels determine Kodaira maps $\Phi_{N}: M \rightarrow P H^{0}\left(M, L^{N}\right)^{\prime}$ to projective space, defined by $\Phi_{N}(z)=\left\{s_{N}: s_{N}(z)=0\right\}$. Equivalently, we can choose an orthonormal basis $S_{1}^{N}, \ldots, S_{d_{N}}^{N}$ of $H^{0}\left(M, L^{N}\right)$ and write

$$
\Phi_{N}: M \rightarrow \mathbb{C P}^{d_{N}-1}, \quad \Phi_{N}(z)=\left(S_{1}^{N}(z): \cdots: S_{d_{N}}^{N}(z)\right) .
$$

We also define the lifts of the Kodaira maps:

$$
\widetilde{\Phi}_{N}: X \rightarrow \mathbb{C}^{d_{N}}, \quad \widetilde{\Phi}_{N}(x)=\left(S_{1}^{N}(x), \ldots, S_{d_{N}}^{N}(x)\right) .
$$


Note that

$$
\Pi_{N}(x, y)=\widetilde{\Phi}_{N}(x) \cdot \overline{\widetilde{\Phi}_{N}(y)}
$$

in particular,

$$
\Pi_{N}(x, x)=\left\|\widetilde{\Phi}_{N}(x)\right\|^{2} .
$$

We will need several results on the diagonal and off-diagonal asymptotics of the Bergman-Szegö kernels. It is proved in [Ca, Ze2] in the holomorphic case and [ShZe2] in the almost-holomorphic case that there exists a complete asymptotic expansion:

$$
\Pi_{N}(z, 0 ; z, 0)=a_{0} N^{m}+a_{1}(z) N^{m-1}+a_{2}(z) N^{m-2}+\ldots
$$

for certain smooth coefficients $a_{j}(z)$ with $a_{0}=\pi^{-m}$. Hence, the maps $\Phi_{N}$ are welldefined for $N \gg 0$. It follows that

$$
\left\|\widetilde{\Phi}_{N}(x)\right\|=\Pi_{N}(x, x)^{\frac{1}{2}}=\pi^{-m / 2} N^{m / 2}+O\left(N^{m / 2-1}\right)=\left(\pi^{-m / 2}+\varepsilon_{N}\right) N^{m / 2},
$$

where $\varepsilon_{N}$ denotes a term satisfying the uniform estimate

$$
\sup _{x \in X}\left|\varepsilon_{N}(x)\right| \leq O\left(\frac{1}{N}\right)
$$

As a further corollary one obtains Tian's almost isometry theorem: Let $\omega_{F S}$ denote the Fubini-Study form on $\mathbb{C P}^{d_{N}-1}$. Then

$$
\left\|\frac{1}{N} \Phi_{N}^{*}\left(\omega_{F S}\right)-\omega\right\|_{\mathcal{C}^{k}}=O\left(\frac{1}{N}\right)
$$

for any $k$.

Off-diagonal asymptotics have been obtained in [ShZe2] and have been studied very precisely in [Ch1]. The results are as follows:

a) Within a $\frac{C}{\sqrt{N}}$ neighborhood of the diagonal, the Bergman-Szegö kernel is given by the scaling asymptotics:

$$
N^{-m} \Pi_{N}\left(z_{0}+u / \sqrt{N}, \theta / N ; z_{0}+v / \sqrt{N}, 0\right) \sim \Pi_{1}^{\mathbf{H}}(u, \theta ; v, 0)[1+O(1 / \sqrt{N})] .
$$

Here

$$
\Pi_{1}^{\mathbf{H}}(u, \theta ; v, \varphi)=\frac{1}{\pi^{m}} e^{i(\theta-\varphi)+i \Im(u \cdot \bar{v})-\frac{1}{2}|u-v|^{2}}
$$

is the Szegö kernel of the reduced Heisenberg group.

b) Whenever $d(z, w) \leq C / N^{1 / 3}$, we have:

$$
\left|\Pi_{N}(z, w)\right| \leq\left(\frac{1}{\pi^{m}}+o(1)\right) N^{m} \exp \left(-\frac{1-\varepsilon}{2} N d(z, w)^{2}\right)+O\left(N^{-\infty}\right) .
$$

c) On all of $M$, we have:

$$
\left|\Pi_{N}(z, w)\right| \leq C N^{m} \exp (-\lambda \sqrt{N} d(z, w)) .
$$


The near-diagonal scaling asymptotics in (a) is just the first two terms of a complete asymptotic expansion. Let $P_{0} \in M$ and choose a Heisenberg coordinate chart about $P_{0}$ in the sense of [ShZe2]. Then [ShZe2, Theorem 3.1]

$$
\begin{aligned}
N^{-m} & \Pi_{N}^{P_{0}}\left(\frac{u}{\sqrt{N}}, \frac{\theta}{N} ; \frac{v}{\sqrt{N}}, \frac{\varphi}{N}\right) \\
& =\Pi_{1}^{\mathbf{H}}(u, \theta ; v, \varphi)\left[1+\sum_{r=1}^{K} N^{-r / 2} b_{r}\left(P_{0}, u, v\right)+N^{-(K+1) / 2} R_{K}\left(P_{0}, u, v, N\right)\right]
\end{aligned}
$$

where $\left\|R_{K}\left(P_{0}, u, v, N\right)\right\|_{\mathcal{C}^{j}(\{|u| \leq \rho,|v| \leq \rho\}} \leq C_{K, j, \rho}$ for $j \geq 0, \rho>0$ and $C_{K, j, \rho}$ is independent of the point $P_{0}$ and choice of coordinates.

The estimate (b) on the larger $N^{-1 / 3}$ balls is from [ShZe2, Lemma 5.2(ii)]. The off-diagonal estimate (c) follows by an Agmon distance argument, as noted by $\mathrm{M}$. Christ [Ch1]; see [Be, Theorem 2.5] for an elementary proof.

3. $\mathcal{L}^{\infty}$ norms: Proof of Theorem 1.1. The proof of Theorem 1.1 is the same in the complex and almost complex ensembles.

Throughout this section we assume that $\left\|s_{N}\right\|_{\mathcal{L}^{2}}=1$. Our aim is to prove:

$$
\nu_{N}\left\{s_{N} \in S H^{0}\left(M, L^{N}\right): \sup _{M}\left|s_{N}\right|>C \sqrt{\log N}\right\}<O\left(\frac{1}{N^{2}}\right),
$$

for some constant $C<+\infty$. (In fact, for any $k>0$, we can bound the probabilities by $O\left(N^{-k}\right)$ by choosing $C$ to be sufficiently large.)

Proof. Recalling (10), we note that

$$
\Pi_{N}(x, y)=\sum_{j=1}^{d_{N}} S_{j}^{N}(x) \overline{S_{j}^{N}(y)}=\left\langle\tilde{\Phi}_{N}(x), \tilde{\Phi}_{N}(y)\right\rangle .
$$

Let $s_{N}=\sum_{j=1}^{d_{N}} c_{j} S_{j}^{N} \quad\left(\sum\left|c_{j}\right|^{2}=1\right)$ denote a random element of $S H^{0}\left(M, L^{N}\right)=$ $S \mathcal{H}_{N}^{2}(X)$, and write $c=\left(c_{1}, \ldots, c_{d_{N}}\right)$. Recall that

$$
s_{N}(x)=\int_{X} \Pi_{N}(x, y) s_{N}(y) d y=\sum_{j=1}^{d_{N}} c_{j} S_{j}^{N}(x)=c \cdot \tilde{\Phi}_{N}(x) .
$$

Thus

$$
\left|s_{N}(x)\right|=\left\|\tilde{\Phi}_{N}(x)\right\| \cos \theta_{x}, \quad \text { where } \cos \theta_{x}=\frac{\left|c \cdot \tilde{\Phi}_{N}(x)\right|}{\left\|\tilde{\Phi}_{N}(x)\right\|} .
$$

(Note that $\theta_{x}$ can be interpreted as the distance in $\mathbb{C P}^{d_{N}-1}$ between $[\bar{c}]$ and $\tilde{\Phi}_{N}(x)$.)

Now fix a point $x \in X$. By Lemma 2.1,

$$
\begin{aligned}
\nu_{N}\left\{s_{N}: \cos \theta_{x} \geq C N^{-m / 2} \sqrt{\log N}\right\} & \leq \exp \left(-\left(d_{N}-1\right) \frac{C^{2} \log N}{N^{m}}\right) \\
& =N^{-C^{2} N^{-m}\left(d_{N}-1\right)} .
\end{aligned}
$$

We can cover $M$ by a collection of $k_{N}$ balls $B\left(z^{j}\right)$ of radius

$$
R_{N}:=\frac{1}{N^{\frac{m+1}{2}}}
$$


centered at points $z^{1}, \ldots, z^{k_{N}}$, where

$$
k_{N} \leq O\left(R^{-2 m}\right) \leq O\left(N^{m(m+1)}\right) .
$$

By (23), we have

$$
\nu_{N}\left\{s_{N} \in S H_{J}^{0}\left(M, L^{N}\right): \max _{j} \cos \theta_{x^{j}} \geq C N^{-m / 2} \sqrt{\log N}\right\} \leq k_{N} N^{-C^{2} N^{-m}\left(d_{N}-1\right)},
$$

where $x^{j}$ denotes a point in $X$ lying above $z^{j}$.

We shall show below that equation (25) together with (14) and (22) implies that the desired sup-norm estimate holds at the centers of the small balls with high probability. To obtain our desired estimate on all of $M$, we first need to extend (25) to points within the balls. To do this, we consider an arbitrary point $w^{j} \in B\left(z^{j}\right)$, and choose points $y^{j} \in X$ lying above the points $w^{j}$. We must estimate the distance, which we denote by $\delta_{N}^{j}$, between $\Phi_{N}\left(z^{j}\right)$ and $\Phi_{N}\left(w^{j}\right)$ in $\mathbb{C} \mathbb{P}^{d_{N}-1}$. Letting $\gamma$ denote the geodesic in $M$ from $z^{j}$ to $w^{j}$, we conclude by (16) that

$$
\begin{aligned}
\delta_{N}^{j} & \leq \int_{\Phi_{N *} \gamma} \sqrt{\omega_{F S}}=\int_{\gamma} \sqrt{\Phi_{N}^{*} \omega_{F S}} \leq \sqrt{N} \int_{\gamma}\left(1+\varepsilon_{N}\right) \sqrt{\omega} \\
& \leq\left(1+\varepsilon_{N}\right) N^{\frac{1}{2}} R_{N}=\frac{1+\varepsilon_{N}}{N^{m / 2}} .
\end{aligned}
$$
$(26)$

By the triangle inequality in $\mathbb{C P}^{d_{N}-1}$, we have $\left|\theta_{x^{j}}-\theta_{y^{j}}\right| \leq \delta_{N}^{j}$. Therefore by

$$
\cos \theta_{x^{j}} \geq \cos \theta_{y^{j}}-\delta_{N}^{j} \geq \cos \theta_{y^{j}}-\frac{1+\varepsilon_{N}}{N^{m / 2}}
$$

By (27),

$$
\cos \theta_{y^{j}} \geq \frac{(C+1) \sqrt{\log N}}{N^{m / 2}} \Rightarrow \cos \theta_{x^{j}} \geq \frac{(C+1) \sqrt{\log N}-\left(1+\varepsilon_{N}\right)}{N^{m / 2}} \geq \frac{C \sqrt{\log N}}{N^{m / 2}}
$$

and thus

$$
\begin{aligned}
& \left\{s_{N} \in S H_{J}^{0}\left(M, L^{N}\right): \sup \cos \theta \geq(C+1) N^{-m / 2} \sqrt{\log N}\right\} \\
& \subset\left\{s_{N} \in S H_{J}^{0}\left(M, L^{N}\right): \max _{j} \cos \theta_{x^{j}} \geq C N^{-m / 2} \sqrt{\log N}\right\} .
\end{aligned}
$$

Hence by (25),

$\nu_{N}\left\{s_{N} \in S H_{J}^{0}\left(M, L^{N}\right): \sup \cos \theta \geq(C+1) N^{-m / 2} \sqrt{\log N}\right\} \leq k_{N} N^{-C^{2} N^{-m}\left(d_{N}-1\right)}$.

It follows from (4), (14), (22) and (28) that

$$
\begin{aligned}
\nu_{N}\left\{s_{N} \in S H_{J}^{0}\left(M, L^{N}\right): \sup _{M}\left|s_{N}\right| \geq(C+2) \sqrt{\log N}\right\} \\
\quad \leq k_{N} N^{-C^{2} N^{-m}\left(d_{N}-1\right)} \leq O\left(N^{m(m+1)-\frac{C^{2}}{m !+1}}\right) .
\end{aligned}
$$

Choosing $C=(m+1) \sqrt{m !+1}$, we obtain the desired estimate. $\square$

REMARK. An alternate proof of this estimate, which does not depend on Tian's theorem, is given by the case $k=0$ of the $\mathcal{C}^{k}$ estimate in $\S 5$. 
3.1. Relation to Levy concentration. The estimate in Theorem 1.1 is very closely related to Levy's estimate. The proof shows that

(i) $\mathcal{L}_{N}^{\infty}$ is Lipschitz continuous with norm $\frac{N^{m / 2}}{\sqrt{\log N}} \leq\left\|\mathcal{L}_{N}^{\infty}\right\|_{L i p} \leq N^{m / 2}$;

(ii) The median of $\mathcal{L}_{N}^{\infty}$ satisfies: $\mathcal{M}_{\mathcal{L}_{N}^{\infty}} \leq C_{m} \sqrt{\log N}$ for sufficiently large $N$.

Indeed, Lipschitz continuity follows from equivalence of norms on finite dimensional vector spaces. To estimate the Lipschitz norm, we recall the well-known fact that the $\mathcal{L}^{2}$-normalized 'coherent states' $\Phi_{N}^{w}(z)=\frac{\Pi_{N}(z, w)}{\sqrt{\Pi_{N}(w, w)}}$ are the global maxima of $\mathcal{L}_{N}^{\infty}$ on $S H^{0}\left(M, L^{N}\right)$, as follows from the Schwartz inequality applied to the reproducing identity $s(z)=\int_{M} \Pi_{N}(z, w) s(w) d V(w)$. Moreover, $\left\|\Phi_{N}^{w}(z)\right\|_{\mathcal{L}^{\infty}}=\sqrt{\Pi_{N}(w, w)} \sim$ $N^{m / 2}$. It follows that

$$
\left|\left\|s_{1}+s_{2}\right\|_{\infty}-\left\|s_{1}\right\|_{\infty}\right| \leq 3 N^{m / 2}
$$

Now let $s_{1}$ have $\mathcal{L}^{\infty}$ norm $\leq C \sqrt{\log N}$ and let $s_{1}=\Phi_{N}^{w}$ for some $w$. Then we see that

$$
\left|\left\|s_{1}+s_{2}\right\|_{\infty}-\left\|s_{1}\right\|_{\infty}\right| \geq \frac{N^{m / 2}}{\sqrt{\log N}}
$$

It obviously follows from (i)-(ii) combined with the Levy estimate (1) that (for any $C>0$ )

$$
\mu\left\{s \in S H^{0}\left(M, L^{N}\right): f_{N}^{\infty}(s) \geq C \sqrt{\log N}\right\} \leq \exp \left(-C\left(d_{N}-1\right) \log N / 2 N^{m}\right) .
$$

Since $d_{N} \sim N^{m}$, this is essentially the same estimate as in Theorem 1.1.

The question arises to find the true order of magnitude of the median $\mathcal{M}_{\mathcal{L}_{N}^{\infty}}$. It would seem to be smaller than $\sqrt{\log N}$.

4. $\mathcal{L}^{p}$ norms: Proof of Theorem 1.3. We now consider $\mathcal{L}^{p}$ norms for $p<\infty$. We denote by $\mathcal{L}_{N}^{p}: S \mathcal{H}_{N} \rightarrow \mathbb{R}^{+}$the functional $\mathcal{L}^{p}\left(s_{N}\right)=\left\|s_{N}\right\|_{\mathcal{L}^{p}}, s_{N} \in S \mathcal{H}_{N}$. Recall that $\mathcal{H}_{N}=H^{0}\left(M, L^{N}\right)$ in the holomorphic case, or more generally, $\mathcal{H}_{N}=H_{J}^{0}\left(M, L^{N}\right)$ in the symplectic case. Theorem 1.3 follows from the Levy concentration of measure inequality (1) applied to estimates of the Lipschitz norm of $\mathcal{L}_{N}^{p}$ and of its median value, which we give in the following two Lemmas.

4.1. Estimate of the Lipschitz norm of $\mathcal{L}_{N}^{p}$. The first step in the proof of Theorem 1.3 is:

LEMma 4.1. The Lipschitz norm of $\mathcal{L}_{N}^{p}$ in dimension $m$ is $O\left(N^{m(1 / 2-1 / p)}\right)$.

Proof. The main point is to show that

$$
\sup _{s_{N} \in S \mathcal{H}_{N}}\left\|s_{N}\right\|_{\mathcal{L}^{p}(M)} \leq C N^{m(1 / 2-1 / p)}
$$

and hence

$$
\left|\left\|s_{N}\right\|_{\mathcal{L}^{p}(M)}-\left\|\tilde{s}_{N}\right\|_{\mathcal{L}^{p}(M)}\right| \leq\left\|s_{N}-\tilde{s}_{N}\right\|_{\mathcal{L}^{p}(M)} \leq C N^{m(1 / 2-1 / p)}\left\|s_{N}-\tilde{s}_{N}\right\|_{\mathcal{L}^{2}(M)} .
$$

To prove (30), it suffices to show the following estimate for the $\mathcal{L}^{p} \rightarrow \mathcal{L}^{q}$ mapping norm of $\Pi_{N}$ :

$$
\left\|\Pi_{N} f\right\|_{\mathcal{L}^{q}(M)} \leq C N^{m(1 / p-1 / q)}\|f\|_{\mathcal{L}^{p}(M)}
$$


We shall apply the Shur-Young inequality which bounds the norm of a symmetric integral operator $K(x, y): \mathcal{L}^{p} \rightarrow \mathcal{L}^{q}$ by

$$
\|K\|_{\mathcal{L}^{p} \rightarrow \mathcal{L}^{q}} \leq C_{p}\left[\sup _{x} \int_{M}|K(x, y)|^{r} d \mu(y)\right]^{1 / r}, \quad \frac{1}{r}=1-\frac{1}{p}+\frac{1}{q} .
$$

We break up the integral

$$
\begin{aligned}
\int_{M}\left|\Pi_{N}(z, w)\right|^{r} d V(w) & =\int_{d(z, w) \leq N^{-1 / 3}}\left|\Pi_{N}(z, w)\right|^{r} d V(w) \\
& +\int_{d(z, w) \geq N^{-1 / 3}}\left|\Pi_{N}(z, w)\right|^{r} d V(w) .
\end{aligned}
$$

For the first term, we have by (17),

$\int_{d(z, w) \leq N^{-1 / 3}}\left|\Pi_{N}(z, w)\right|^{r} d V(w) \leq C N^{m r} \int_{\mathbb{C}^{m}} e^{-r N|u|^{2} / 4} d u+O\left(N^{-\infty}\right) \leq C^{\prime} N^{m r-m}$.

The second term is rapidly decaying. Indeed by (18),

$$
\int_{d(z, w) \geq N^{-1 / 3}}\left|\Pi_{N}(z, w)\right|^{r} d V(w) \leq O\left(N^{-\infty}\right)
$$

and hence

$$
\int_{M}\left|\Pi_{N}(z, w)\right|^{r} d V(w)=O\left(N^{m r-m}\right) .
$$

We then obtain (31) from the Shur-Young inequality and (32).

REMARK. Lemma 4.1 is sharp. Indeed, we have

$$
\sup _{s_{N} \in \mathcal{H}_{N}} \frac{\left\|s_{N}\right\|_{\mathcal{L}^{p}(M)}}{\left\|s_{N}\right\|_{\mathcal{L}^{2}(M)}} \sim N^{m(1 / 2-1 / p)}
$$

To prove the lower bound of (33), we let $s_{N}$ be the coherent state

$$
\Phi_{N}^{w}(z):=\frac{\Pi_{N}(z, w)}{\left\|\Pi_{N}(\cdot, w)\right\|}=N^{-m / 2} \Pi_{N}(z, w) .
$$

We have:

$$
\left\|\Phi_{N}^{w}\right\|_{\mathcal{L}^{p}} \sim N^{-m / 2}\left[\int_{M}\left|\Pi_{N}(z, w)\right|^{p} d V\right]^{1 / p} \sim N^{-m / 2} N^{m(1-1 / p)}=N^{m(1 / 2-1 / p)}
$$

4.2. Estimate of the median. Unlike the case of the sup norm, we can estimate the median directly by using Chebychev's inequality.

LEMma 4.2. Let $\mathcal{M}_{\mathcal{L}_{N}^{p}}$ denote the median of $\mathcal{L}_{N}^{p}$. Then there is a constant $\alpha=\alpha(m, p)$ such that

$$
\mathcal{M}_{\mathcal{L}_{N}^{p}} \leq \alpha \quad \forall N \geq 1
$$


Proof. By Chebychev, we have

$$
\begin{aligned}
\nu_{N}\left\{s_{N} \in S \mathcal{H}_{N}: \mathcal{L}_{N}^{p}\left(s_{N}\right)>t\right\} & \leq \frac{1}{t^{p}} \mathbf{E}\left(\left(\mathcal{L}_{N}^{p}\right)^{p}\right) \\
& =\frac{1}{t^{p}} \int_{S^{2 d_{N}-1}} \int_{X}\left|\sum_{j=1}^{d_{N}} c_{j} S_{j}^{N}(x)\right|^{p} d V(x) d \nu_{N}(c) .
\end{aligned}
$$

Let us write

$$
S_{j}^{N}(x)=\Pi_{N}(x, x)^{\frac{1}{2}} u_{j}(x)=\left\|\tilde{\Phi}_{N}(x)\right\| u_{j}(x),
$$

so that $\sum_{j=1}^{d_{N}}\left|u_{j}(x)\right|^{2} \equiv 1$. We then have by (14),

$$
\begin{aligned}
\int_{S^{2 d_{N}-1}} \int_{X}\left|\sum_{j=1}^{d_{N}} c_{j} S_{j}^{N}(x)\right|^{p} d V(x) d \nu_{N}(c) \\
=\int_{X} \Pi_{N}(x, x)^{p / 2} \int_{S^{2 d_{N}-1}}\left|\sum_{j=1}^{d_{N}} c_{j} u_{j}(x)\right|^{p} d \nu_{N}(c) d V(x) \\
=A_{p, d_{N}} \int_{X} \Pi_{N}(x, x)^{p / 2} d V(x)=\left[C_{m} A_{p, d_{N}}+o(1)\right] N^{m p / 2}
\end{aligned}
$$

where

$$
A_{p, d}=\int_{S^{2 d-1}}\left|w_{1}\right|^{p} d \mu(w) \quad\left(\mu\left(S^{2 d-1}\right)=1\right)
$$

and $C_{m}$ depends only on $m$. To compute $A_{p, d}$, we evaluate the integral:

$$
\begin{aligned}
\frac{1}{\pi^{d}} \int_{\mathbb{C}^{d}}\left|z_{1}\right|^{p} e^{-\|z\|^{2}} d z & =\frac{1}{\pi} \int_{\mathbb{C}}\left|z_{1}\right|^{p} e^{-\left|z_{1}\right|^{2}} d z_{1}=\Gamma\left(\frac{p}{2}+1\right) \\
& =\frac{\sigma_{d}}{\pi^{d}} \int_{0}^{\infty} \int_{S^{2 d-1}}\left|w_{1}\right|^{p} r^{p} e^{-r^{2}} r^{2 d-1} d \mu(w) d r \\
& =A_{p, d} \frac{\sigma_{d}}{\pi^{d}} \int_{0}^{\infty} r^{p+2 d-1} e^{-r^{2}} d r \\
& =A_{p, d} \frac{\Gamma\left(d+\frac{p}{2}\right)}{\Gamma(d)} .
\end{aligned}
$$

Therefore

$$
A_{p, d}=\Gamma\left(\frac{p}{2}+1\right) \frac{\Gamma(d)}{\Gamma\left(d+\frac{p}{2}\right)}=\left[\Gamma\left(\frac{p}{2}+1\right)+o(1)\right] d^{-p / 2}
$$

Recalling that $d_{N}=\frac{c_{1}(L)^{m}}{m !} N^{m}+\cdots$, we then have

$$
\nu_{N}\left\{s_{N} \in S \mathcal{H}_{N}: \mathcal{L}_{N}^{p}\left(s_{N}\right)>t\right\} \leq \frac{1}{t^{p}} C_{m, p},
$$

for some constant $C_{m, p}$ depending only on $m$ and $p$. Substituting $t=\mathcal{M}_{\mathcal{L}_{N}^{p}}$ into (36) so that the left side equals $\frac{1}{2}$, we conclude that the sequence $\mathcal{M}_{\mathcal{L}_{N}^{p}}$ of medians is bounded. 
5. $\mathcal{C}^{k}$ norms: Proof of Theorem 1.4. Our first aim is to prove the estimate

$$
\left\|\nabla^{k} s_{N}\right\|_{\infty} /\left\|s_{N}\right\|_{2}=O\left(\sqrt{N^{k} \log N}\right) \text { almost surely }
$$

on complex and almost complex manifolds. The proof follows the pattern of the above sup-norm estimate.

We pause to summarize and review our notation in [ShZe2, ShZe3] for the various differential operators that we use in the complex case:

a) Derivatives on $M$ :

$$
\begin{aligned}
\text { - } & \frac{\partial}{\partial z_{j}}=\frac{1}{2} \frac{\partial}{\partial x_{j}}-\frac{i}{2} \frac{\partial}{\partial y_{j}}, \quad \frac{\partial}{\partial \bar{z}_{j}}=\frac{1}{2} \frac{\partial}{\partial x_{j}}+\frac{i}{2} \frac{\partial}{\partial y_{j}} \\
\text { - } & Z_{j}^{M}=\frac{\partial}{\partial z_{j}}+\sum \bar{B}_{j k}(z) \frac{\partial}{\partial \bar{z}_{k}}, \bar{Z}_{j}^{M}=\frac{\partial}{\partial \bar{z}_{j}}+\sum B_{j k}(z) \frac{\partial}{\partial z_{k}}, B_{j k}\left(P_{0}\right)=0 \\
& \left\{Z_{1}, \ldots, Z_{m}\right\} \text { is a local frame for } T^{1,0} M
\end{aligned}
$$

b) Derivatives on $X$ :

- $\frac{\partial^{h}}{\partial z_{j}}=\frac{\partial}{\partial z_{j}}-A_{j}(z) \frac{\partial}{\partial \theta}=$ horizontal lift of $\frac{\partial}{\partial z_{j}}, A_{j}\left(P_{0}\right)=0$

- $Z_{j}=$ horizontal lift of $Z_{j}^{M}$;

- $d^{h}=\partial_{b}+\bar{\partial}_{b}=$ horizontal exterior derivative on $X$.

c) Covariant derivatives on $M$ :

- $\nabla: \mathcal{C}^{\infty}\left(M, L^{N} \otimes\left(T^{*} M\right)^{\otimes k}\right) \rightarrow \mathcal{C}^{\infty}\left(M, L^{N} \otimes\left(T^{*} M\right)^{\otimes(k+1)}\right)$;

- $\nabla^{k}=\nabla \circ \cdots \circ \nabla: \mathcal{C}^{\infty}\left(M, L^{N}\right) \rightarrow \mathcal{C}^{\infty}\left(M, L^{N} \otimes\left(T^{*} M\right)^{\otimes k}\right)$;

- $\nabla=\partial+\bar{\partial}, \quad \bar{\partial}: \mathcal{C}^{\infty}\left(M, L^{N}\right) \rightarrow \mathcal{C}^{\infty}\left(M, L^{N} \otimes T^{* 0,1} M\right)$.

d) Derivatives on $X \times X$ :

- $d_{j}^{1}, d_{j}^{2}$ : the operator $\frac{\partial^{h}}{\partial z_{j}}$ applied to the first and second factors, respectively;

- $Z_{j}^{1}, Z_{j}^{2}$ : the operator $Z_{j}$ applied to the first and second factors, respectively.

5.1. Derivatives of holomorphic and almost holomorphic sections. To prove (37), we first note a consequence (Lemma 5.2) of our near-diagonal asymptotics. Recall that a differential operator on $X$ is horizontal if it is generated by horizontal vector fields. In particular the operators $\nabla^{k}: \mathcal{C}^{\infty}\left(M, L^{N}\right) \rightarrow \mathcal{C}^{\infty}\left(M, L^{N} \otimes\left(T^{*} M\right)^{\otimes k}\right)$ are given by (vector valued) horizontal differential operators (independent of $N$ ) on $X$. By definition, horizontal differential operators on $X \times X$ are generated by the horizontal differential operators on the first and second factors. We begin with the following estimate:

Lemma 5.1. Let $P_{k}$ be a horizontal differential operator of order $k$ on $X \times X$. Then

$$
\left.P_{k} \Pi_{N}(x, y)\right|_{x=y}=O\left(N^{m+k / 2}\right) .
$$

Proof. Let $x_{0}=\left(P_{0}, 0\right)$ be an arbitrary point of $X$, and choose local real 'Heisenberg' coordinates $\left(x_{1}, \ldots, x_{2 m}, \theta\right)$ about $\left(P_{0}, 0\right)$ as in the hypothesis of Theorem 3.1 of [ShZe2] (with $z_{q}=x_{q}+i x_{m+q}$ ). We let $\frac{\partial^{h}}{\partial x_{q}}$ denote the horizontal lift of $\frac{\partial}{\partial x_{q}}$ to $X$ :

$$
\frac{\partial^{h}}{\partial x_{q}}=\frac{\partial}{\partial x_{q}}-\tilde{A}_{q}(x) \frac{\partial}{\partial \theta}, \quad \tilde{A}_{q}=\left(\alpha, \frac{\partial}{\partial x_{q}}\right) .
$$


Since $\left.\frac{\partial}{\partial x_{q}}\right|_{x_{0}}$ is assumed to be horizontal, we have $\tilde{A}_{q}\left(P_{0}\right)=0$.

We let $d_{q}^{1}, d_{q}^{2}$ denote the operator $\frac{\partial^{h}}{\partial x_{q}}$ applied to the first and second factors, respectively, on $X \times X$. For this result, we need only the zeroth order estimate of (19):

$$
\Pi_{N}\left(\frac{u}{\sqrt{N}}, \frac{s}{N} ; \frac{v}{\sqrt{N}}, \frac{t}{N}\right)=N^{m} e^{i(s-t)+\psi_{2}(u, v)} \mathcal{R}\left(P_{0}, u, v, N\right)
$$

where $\mathcal{R}\left(P_{0}, u, v, N\right)$ denotes a term satisfying the remainder estimate of (19):

$$
\left\|\mathcal{R}\left(P_{0}, u, v, N\right)\right\|_{\mathcal{C}^{j}(\{|u| \leq \rho,|v| \leq \rho\}} \leq C_{j, \rho}
$$

for $j \geq 0, \rho>0$, where $C_{j, \rho}$ is independent of the point $P_{0}$ and choice of coordinates. Here,

$$
\psi_{2}(u, v)=u \cdot \bar{v}-\frac{1}{2}\left(|u|^{2}+|v|^{2}\right)
$$

Differentiating (38) and noting that $\partial / \partial x_{q}=\sqrt{N} \partial / \partial u_{q}, \partial / \partial \theta=N \partial / \partial s$, we have

$$
\begin{aligned}
& d_{q}^{1} \Pi_{N}\left(\frac{u}{\sqrt{N}}, \frac{s}{N} ; \frac{v}{\sqrt{N}}, \frac{t}{N}\right) \\
& =\sqrt{N}\left(\frac{\partial}{\partial u_{q}}-\sqrt{N} \tilde{A}_{q}\left(P_{0}+\frac{u}{\sqrt{N}}\right) \frac{\partial}{\partial s}\right)\left(N^{m} e^{i(s-t)+\psi_{2}(u, v)} \mathcal{R}\right) \\
& =N^{m+1 / 2} e^{i(s-t)+\psi_{2}(u, v)}\left\{\left[L_{q}(u, v)-i \sqrt{N} \tilde{A}_{q}\left(\frac{u}{\sqrt{N}}\right)\right]+\frac{\partial}{\partial u_{q}} \mathcal{R}\right\} \\
& =N^{m+1 / 2} e^{i(s-t)+\psi_{2}(u, v)} \widetilde{\mathcal{R}}=O\left(N^{m+1 / 2}\right)
\end{aligned}
$$

where $L_{q}:=\frac{\partial \psi_{2}}{\partial u_{q}}$ is a linear function. The same estimate holds for $d_{q}^{2} \Pi_{N}$. Indeed, the above computation yields:

$$
d_{q}^{j} e^{i(s-t)+\psi_{2}(u, v)} \mathcal{R}\left(P_{0}, u, v, N\right)=\sqrt{N} e^{i(s-t)+\psi_{2}(u, v)} \widetilde{\mathcal{R}}\left(P_{0}, u, v, N\right)
$$

for $j=1,2, q=1, \ldots, 2 m$. The desired estimate follows by iterating (41).

REmark. The assumption that $P_{k}$ is horizontal in Lemma 5.1 is necessary, since the operator $\partial / \partial \theta$ multiplies the estimate by $N$ instead of $\sqrt{N}$.

Lemma 5.2. Let $P_{k}$ be a horizontal differential operator of order $k$ on $X$. Then

$$
\sup _{X}\left\|P_{k} \tilde{\Phi}_{N}\right\|=O\left(N^{\frac{m+k}{2}}\right) .
$$

Proof. Let $P_{k}^{1}, P_{k}^{2}$ denote the operator $P_{k}$ applied to the first and second factors, respectively, on $X \times X$. Differentiating (20) and restricting to the diagonal, we obtain

$$
P_{k}^{1} \bar{P}_{k}^{2} \Pi_{N}(x, x)=\left\|P_{k} \tilde{\Phi}_{N}(x)\right\|^{2} .
$$

The conclusion follows from (42) and Lemma 5.1 applied to the horizontal differential operator (of order $2 k$ ) $P_{k}^{1} \bar{P}_{k}^{2}$ on $X \times X$. 
We are now ready to use the small-ball method of the previous section to show that $\left\|\nabla^{k} s_{N}\right\|_{\infty} /\left\|s_{N}\right\|_{\mathcal{L}^{2}}=O\left(\sqrt{N^{k} \log N}\right)$ almost surely. It is sufficient to show that (2) holds for $j=2$, i.e.,

$$
\nu_{N}\left\{s_{N} \in S H_{J}^{0}\left(M, L^{N}\right): \sup _{M}\left|\nabla^{k} s_{N}\right|>C \sqrt{N^{k} \log N}\right\}<O\left(\frac{1}{N^{2}}\right),
$$

for $C$ sufficiently large. To verify (2), we may regard $s_{N}$ as a function on $X$ and replace $\nabla^{k}$ by a horizontal $r_{\theta}$-invariant differential operator of order $k$ on $X$. we have

As before, we let $s_{N}=\sum c_{j} S_{j}^{N}$ denote a random element of $S \mathcal{H}_{N}^{2}(X)$. By (21),

$$
P_{k} s_{N}(x)=\int_{X} P_{k}^{1} \Pi_{N}(x, y) s_{N}(y) d y=\sum_{j=1}^{d_{N}} c_{j} P_{k} S_{j}^{N}(x)=c \cdot P_{k} \tilde{\Phi}_{N}(x) .
$$

We then have

$$
\left|P_{k} s_{N}(x)\right|=\left\|P_{k} \tilde{\Phi}_{N}(x)\right\| \cos \theta_{x}, \quad \text { where } \cos \theta_{x}=\frac{\left|c \cdot P_{k} \tilde{\Phi}_{N}(x)\right|}{\left\|P_{k} \tilde{\Phi}_{N}(x)\right\|} .
$$

Now fix a point $x \in X$. As before, (23) holds, and hence by Lemma 5.2 we have

$$
\nu_{N}\left\{s_{N} \in S \mathcal{H}_{N}^{2}:\left|P_{k} s_{N}(x)\right| \geq C^{\prime} \sqrt{N^{k} \log N}\right\} \leq k_{N} N^{-C^{2} N^{-m}\left(d_{N}-1\right)}
$$

where $C^{\prime}=C \sup _{N, x} N^{-(m+k) / 2}\left|P_{k} \tilde{\Phi}_{N}(x)\right|$.

We again cover $M$ by a collection of $k_{N}$ very small balls $B\left(z^{j}\right)$ of radius $R_{N}=$ $N^{-\frac{m+1}{2}}$ and first show that the probability of the required condition holding at the centers of all the balls is small. Choosing points $x^{j} \in X$ lying above the centers $z^{j}$ of the balls, we then have

$$
\nu_{N}\left\{s_{N} \in S \mathcal{H}_{N}^{2}: \max _{j}\left|P_{k} s_{N}\left(x_{j}\right)\right| \geq C^{\prime} \sqrt{N^{k} \log N}\right\} \leq k_{N} N^{-C^{2} N^{-m}\left(d_{N}-1\right)} .
$$

Now suppose that $w^{j}$ is an arbitrary point in $B\left(z^{j}\right)$, and let $y^{j}$ be the point of $X$ above $w^{j}$ such that the horizontal lift of the geodesic from $z^{j}$ to $w^{j}$ connects $x^{j}$ and $y^{j}$. Hence by Lemma 5.2, we have

$$
\left\|P_{k} \tilde{\Phi}_{N}\left(x^{j}\right)-P_{k} \tilde{\Phi}_{N}\left(y^{j}\right)\right\| \leq \sup _{M}\left\|d^{h}\left(P_{k} \tilde{\Phi}_{N}\right)\right\| r_{N}=O\left(N^{\frac{m+k+1}{2}}\right) r_{N}=O\left(N^{\frac{k}{2}}\right) .
$$

It follows as before from (47) and (48) that

$$
\begin{aligned}
\nu_{N}\left\{s_{N} \in S \mathcal{H}_{N}^{2}: \sup _{X}\left|P_{k} s_{N}\right|\right. & \left.\geq\left(C^{\prime}+1\right) \sqrt{N^{k} \log N}\right\} \\
\leq & k_{N} N^{-C^{2} N^{-m}\left(d_{N}-1\right)} \leq O\left(N^{m(m+1)-\frac{C^{2}}{m !+1}}\right) .
\end{aligned}
$$

(Here, we used the fact that $\left|P_{k} s_{N}\right|$ is constant on the fibers of $\pi: X \rightarrow M$.) Thus, (2) holds with $C$ sufficiently large.

5.2. $\bar{\partial}$ derivatives of almost holomorphic sequences. In this section we obtain additional results on the complex derivatives of almost holomorphic sections. The results are of course trivial in the holomorphic case. As mentioned in the introduction, they are relevant to the use of asymptotically holomorphic sections in almost complex geometry. 
5.2.1. The estimate $\left\|\bar{\partial} s_{N}\right\|_{\infty} /\left\|s_{N}\right\|_{2}=O(\sqrt{\log N})$ almost surely. The proof of the $\bar{\partial} s_{N}$ estimate follows the pattern of the above estimate. However, there is one crucial difference: we must show the following upper bound for the modulus of $\bar{\partial}_{b} \tilde{\Phi}_{N}$. This estimate is a factor of $\sqrt{N}$ better than the one for $d^{h} \tilde{\Phi}_{N}$ arising from Lemma 5.2 ; the proof depends on the precise second order approximation of Theorem 3.1 of [ShZe2] (see (19)).

LEMMA 5.3. $\sup _{X}\left\|\bar{\partial}_{b} \tilde{\Phi}_{N}(x)\right\| \leq O\left(N^{m / 2}\right)$.

Proof. Let $x_{0}=\left(P_{0}, 0\right)$ be an arbitrary point of $X$, and choose preferred local coordinates $\left(z_{1}, \ldots, z_{m}, \theta\right)$ about $\left(P_{0}, 0\right)$ as in the hypothesis of Theorem 19. We lift a local frame $\left\{\bar{Z}_{q}^{M}\right\}$ to obtain the local frame $\left\{\bar{Z}_{1}, \ldots, \bar{Z}_{m}\right\}$ for $H^{0,1} X$ given by

$$
\bar{Z}_{q}=\frac{\partial^{h}}{\partial \bar{z}_{q}}+\sum_{r=1}^{m} B_{q r}(z) \frac{\partial^{h}}{\partial z_{r}}, \quad B_{q r}\left(P_{0}\right)=0
$$

It suffices to show that

$$
N^{-m / 2}\left|\bar{Z}_{q} \tilde{\Phi}_{N}\left(x_{0}\right)\right| \leq C
$$

where $C$ is a constant independent of $x_{0}$.

By (19), we have

$$
\begin{aligned}
& N^{-m} \Pi_{N}\left(\frac{u}{\sqrt{N}}, \frac{s}{N} ; \frac{v}{\sqrt{N}}, \frac{t}{N}\right) \\
& =\frac{1}{\pi^{m}} \varphi_{0}(u, s) \overline{\varphi_{0}(v, t)} e^{u \cdot \bar{v}}\left[1+\frac{1}{\sqrt{N}} b_{1}\left(P_{0}, u, v\right)+\frac{1}{N} R_{2}\left(P_{0}, u, v, N\right)\right],
\end{aligned}
$$

where

$$
\varphi_{0}(z, \theta)=e^{i \theta-|z|^{2} / 2} .
$$

(The function $\varphi_{0}$ is the 'ground state' for the 'annihilation operators' $\bar{Z}_{q}$ in the Heisenberg model; see the remark in [BSZ2, §1.3.2]). In our case, $\bar{Z}_{q} \varphi_{0}$ does not vanish as in the model case, but instead satisfies the asymptotic bound (53) below.)

We have (see [ShZe2]),

$$
\frac{\partial^{h}}{\partial \bar{z}_{q}}=\frac{\partial}{\partial \bar{z}_{q}}+\left[-\frac{i}{2} z_{q}-R_{1}^{\bar{A}_{q}}(z)\right] \frac{\partial}{\partial \theta}
$$

where $R_{1}^{\bar{A}_{q}}(z)=O\left(|z|^{2}\right)$. Recalling that $z=u / \sqrt{N}, \theta=s / N$, we note that $\varphi_{0}(u, s)=$ $e^{i N \theta-N|z|^{2} / 2}=\varphi_{0}(z, \theta)^{N}$, and thus by (52),

$$
\frac{\partial^{h}}{\partial \bar{z}_{q}} \varphi_{0}(u, s)=\frac{\partial^{h}}{\partial \bar{z}_{q}} e^{i N \theta-N|z|^{2} / 2}=-i N R_{1}^{\bar{A}_{q}}\left(\frac{u}{\sqrt{N}}\right) \varphi_{0}(u, s)=\mathcal{R}\left(P_{0}, u, N\right) \varphi_{0}(u, s),
$$

where as before $\mathcal{R}$ denotes a term satisfying the remainder estimate of (19).

We let $Z_{q}^{1}, Z_{q}^{2}$ denote the operator $Z_{q}$ applied to the first and second factors, respectively, on $X \times X$; we similarly let $d_{q}^{1}, d_{q}^{2}$ denote the operator $\frac{\partial^{h}}{\partial z_{q}}$ applied to the factors of $X \times X$. Equation (42) tells us that

$$
\left\|\bar{Z}_{q} \tilde{\Phi}_{N}(x)\right\|^{2}=\bar{Z}_{q}^{1} Z_{q}^{2} \Pi_{N}(x, x) .
$$


By (49),

$$
\bar{Z}_{q}^{1} Z_{q}^{2}=\left(\overline{d_{q}^{1}}+\sum_{r=1}^{m} B_{q r}(z) d_{r}^{1}\right)\left(d_{q}^{2}+\sum_{\rho=1}^{m} \bar{B}_{q \rho}(w) \overline{d_{\rho}^{2}}\right)
$$

where we recall that $B_{q r}\left(P_{0}\right)=0$.

Differentiating (51), again noting that $\partial / \partial z_{q}=\sqrt{N} \partial / \partial u_{q}, \partial / \partial w_{q}=\sqrt{N} \partial / \partial v_{q}$ and using (53), we obtain

$$
\begin{aligned}
& N^{-m}\left(\overline{d_{q}^{1}} d_{q}^{2} \Pi_{N}\right)\left(\frac{u}{\sqrt{N}}, \frac{s}{N} ; \frac{v}{\sqrt{N}}, \frac{t}{N}\right) \\
& \quad=\frac{1}{\pi^{m}} \varphi_{0}(u, s) \overline{\varphi_{0}(v, t)} e^{u \cdot \bar{v}}\left[\sqrt{N} \frac{\partial^{2}}{\partial \bar{u}_{q} \partial v_{q}} b_{1}+\widetilde{\mathcal{R}}\right] .
\end{aligned}
$$

Since $b_{1}$ has no terms that are quadratic in $(u, \bar{u}, v, \bar{v})$, it follows from (55)-(56) that

$$
\begin{aligned}
& N^{-m}\left|\bar{Z}_{q}^{1} Z_{q}^{2} \Pi_{N}\left(P_{0}, 0 ; P_{0}, 0\right)\right| \\
& \quad=N^{-m}\left|\overline{d_{q}^{1}} d_{q}^{2} \Pi_{N}\left(P_{0}, 0 ; P_{0}, 0\right)\right|=\frac{1}{\pi^{m}}\left|\widetilde{\mathcal{R}}\left(P_{0}, 0,0, N\right)\right| \leq O(1) .
\end{aligned}
$$

The desired estimate (50) now follows immediately from (54) and (57).

By covering $M$ with small balls and repeating the argument of the previous section, using Lemma 5.3, we conclude that

$$
\nu_{N}\left\{s_{N} \in S H_{J}^{0}\left(M, L^{N}\right): \sup _{M}\left|\bar{\partial} s_{N}\right|>C \sqrt{\log N}\right\}<O\left(\frac{1}{N^{2}}\right) .
$$

Thus $\left\|\bar{\partial} s_{N}\right\|_{\infty} /\left\|s_{N}\right\|_{2}=O(\sqrt{\log N})$ almost surely.

5.2.2. The estimate $\left\|\nabla^{k} \bar{\partial} s_{N}\right\|_{\infty} /\left\|s_{N}\right\|_{2}=O\left(\sqrt{N^{k} \log N}\right)$ almost surely. To obtain this final estimate of Theorem 1.4, it suffices to verify the probability estimate

$$
\nu_{N}\left\{s_{N} \in S H_{J}^{0}\left(M, L^{N}\right): \sup _{M}\left|\nabla^{k} \bar{\partial} s_{N}\right|>C \sqrt{N^{k} \log N}\right\}<O\left(\frac{1}{N^{2}}\right) .
$$

Equation (59) follows by again repeating the argument of $\S 3$, using the following lemma. Then

Lemma 5.4. Let $P_{k}$ be a horizontal differential operator of order $k$ on $X(k \geq 0)$.

$$
\sup _{X}\left|P_{k} \bar{\partial}_{b} \tilde{\Phi}_{N}\right|=O\left(N^{\frac{m+k}{2}}\right)
$$

Proof. It suffices to show that

$$
\sup _{U}\left|P_{k} \bar{Z}_{q}^{k} \tilde{\Phi}_{N}\right|=O\left(N^{\frac{m+k}{2}}\right)
$$

for a local frame $\left\{\bar{Z}_{q}\right\}$ of $T^{0,1} M$ over $U$. As before, we have

$$
P_{k}^{1} \bar{P}_{k}^{2} \bar{Z}_{q}^{1} Z_{q}^{1} \Pi_{N}(x, x)=\left|P_{k} \bar{Z}_{q}^{h} \tilde{\Phi}_{N}(x)\right|^{2} .
$$


We claim that

$$
N^{-m} \bar{Z}_{q}^{1} Z_{q}^{2} \Pi_{N}=\frac{1}{\pi^{m}} e^{i(s-t)+\psi_{2}(u, v)}\left[\sqrt{N} \frac{\partial^{2}}{\partial \bar{u}_{q} \partial v_{q}} b_{1}+\mathcal{R}\left(P_{0}, u, v, N\right)\right] .
$$

To obtain the estimate (62), we recall from (55) in the proof of Lemma 5.3 that

$$
\bar{Z}_{q}^{1} Z_{q}^{2}=\overline{d_{q}^{1}} d_{q}^{2}+\sum_{\rho=1}^{m} \bar{B}_{q \rho}(w) \overline{d_{q}^{1} d_{\rho}^{2}}+\sum_{r=1}^{m} B_{q r}(z) d_{r}^{1} d_{q}^{2}+\sum_{r, \rho} B_{q r}(z) \bar{B}_{q \rho}(w) d_{r}^{1} \overline{d_{\rho}^{2}} .
$$

Equation (56) says that the first term of $N^{-m} \bar{Z}_{q}^{1} Z_{q}^{2} \Pi_{N}$ coming from the expansion (63) satisfies the estimate of (62). To obtain the estimate for the second term, we compute:

$$
\begin{aligned}
& N^{-m} \overline{d_{\rho}^{2}} \Pi_{N}\left(\frac{u}{\sqrt{N}}, \frac{s}{N} ; \frac{v}{\sqrt{N}}, \frac{t}{N}\right)=\frac{\sqrt{N}}{\pi^{m}} e^{i(s-t)+\psi_{2}(u, v)} \\
& \quad \cdot\left(\left[\frac{\partial \psi_{2}}{\partial \bar{v}_{\rho}}+i \sqrt{N} A_{\rho}\left(\frac{v}{\sqrt{N}}\right)\right]\left[1+\frac{1}{\sqrt{N}} b_{1}+\frac{1}{N} R_{2}\right]+\frac{1}{\sqrt{N}} \frac{\partial b_{1}}{\partial \bar{v}_{\rho}}+\frac{1}{N} \frac{\partial R_{2}}{\partial \bar{v}_{\rho}}\right) \\
& =\frac{\sqrt{N}}{\pi^{m}} \varphi_{0}(u, s) \overline{\varphi_{0}(v, t)} e^{u \cdot \bar{v}}\left[\frac{\partial \psi_{2}}{\partial \bar{v}_{\rho}}+L_{\rho}(v)+\frac{1}{\sqrt{N}} \tilde{\mathcal{R}}\right]
\end{aligned}
$$

where $L_{\rho}$ is a linear function. Since $\partial^{2} \psi_{2} / \partial \bar{u}_{q} \partial \bar{v}_{\rho} \equiv 0$, it then follows that

$$
N^{-m} \overline{d_{q}^{1} d_{\rho}^{2}} \Pi_{N}=\frac{\sqrt{N}}{\pi^{m}} e^{i(s-t)+\psi_{2}(u, v)} \frac{\partial}{\partial \bar{u}_{q}} \widetilde{\mathcal{R}}\left(P_{0}, u, v, N\right) .
$$

The estimate (62) for the second term follows from (65), using the fact that $B_{q \rho}\left(\frac{v}{\sqrt{N}}\right)=\frac{1}{\sqrt{N}} L_{q \rho}(v)+\cdots$. The proofs of the estimate for the third and fourth terms are similar.

The desired estimate (60) follows as before from (61), (62), and (41), using the fact that $\frac{\partial^{2}}{\partial \bar{u}_{q} \partial v_{q}} b_{1}$ is linear.

\section{REFERENCES}

[Be] B. BERndtsson, Bergman kernels related to Hermitian line bundles over compact complex manifolds, Explorations in Complex and Riemannian Geometry, Contemp. Math., 332, Amer. Math. Soc., Providence, RI, 2003.

[BSZ1] P. BLEHER, B. SHIFFMAN AND S. ZELDITCH, Universality and scaling of correlations between zeros on complex manifolds, Invent. Math., 142 (2000), pp. 351-395.

[BSZ2] P. BLEHER, B. SHIFFMAN AND S. ZELDITCH, Universality and scaling of zeros on symplectic manifolds, Random matrix models and their applications, pp. 31-69, Math. Sci. Res. Inst. Publ., 40, Cambridge Univ. Press, Cambridge, 2001.

[Bo1] J. Bourgain, Applications of the spaces of homogeneous polynomials to some problems on the ball algebra, Proc. Amer. Math. Soc., 93 (1985), pp. 277-283.

[Bo2] J. BourGAIN, Homogeneous polynomials on the ball and polynomial bases, Israel J. Math., 68 (1989), pp. 327-347.

[BG] L. Boutet De Monvel And V. Guillemin, The Spectral Theory of Toeplitz Operators, Ann. Math. Studies 99, Princeton Univ. Press, 1981.

[Ca] D. CATLIN, The Bergman kernel and a theorem of Tian, Analysis and geometry in several complex variables, pp. 1-23, Trends Math., Birkhäuser Boston, Boston, MA, 1999.

[Ch1] M. Christ, On the $\bar{\partial}$ equation in weighted $L^{2}$ norms in $\mathbf{C}^{1}$, J. Geometric Anal., 1 (1991), pp. 193-230. 
[Ch2] M. CHRIST, Slow off-diagonal decay for Szegö kernels associated to smooth Hermitian line bundles, Harmonic analysis at Mount Holyoke (South Hadley, MA, 2001), pp. 77-89, Contemp. Math., 320, Amer. Math. Soc., Providence, RI, 2003.

[Do] S. Donaldson, Symplectic submanifolds and almost complex geometry, J. Diff. Geom., 44 (1996), pp. 666-705.

[Ka] J.-P. Kahane, Some Random Series of Functions, Cambridge Stud. Adv. Math., vol. 5, Cambridge, 1985.

[Le] M. Ledoux, The Concentration of Measure Phenomenon, Math. Surveys and Mono. 89, Amer. Math. Soc., Providence, RI, 2001.

[LMS] A. E. Litvak, V. D. Milman AND G. Schechtman, Averages of norms and quasi-norms, Math. Ann., 312 (1998), pp. 95-124.

[Ne] J. NEuHEISEL, The asymptotic distribution of nodal sets on spheres, Ph.D. thesis, Johns Hopkins University (2000).

[NV] S. Nonnenmacher AND A. Voros, Chaotic eigenfunctions in phase space, J. Stat. Phys., 92 (1998), pp. 431-518.

[PWZ] R. E. PAley, N. Wiener AND Z. Zygmund, Notes on random functions, Math. Z., 37 (1932), pp. 647-68.

[SaZy] R. SAlem AND A. Zygmund, Some properties of trigonometric series whose terms have random signs, Acta Math., 91 (1954), pp. 254-301.

[ShZe1] B. ShIFFMAN AND S. ZELDITCH, Distribution of zeros of random and quantum chaotic sections of positive line bundles, Comm. Math. Phys., 200 (1999), pp. 661-683.

[ShZe2] B. ShIFFMAn And S. ZELDITCH, Asymptotics of almost holomorphic sections of ample line bundles on symplectic manifolds, J. Reine Angew. Math., 544 (2002), pp. 181-222.

[ShZe3] B. ShIFFMAN AND S. ZELDITCH, Asymptotics of almost holomorphic sections of ample line bundles on symplectic manifolds: An addendum, Proc. Amer. Math. Soc., 131 (2003), pp. 291-302.

[Va] J. VANDERKAM, $L^{\infty}$ norms and quantum ergodicity on the sphere, Internat. Math. Res. Notices, 1997, pp. 329-347.

[Ze1] S. ZeLDITCH, A random matrix model for quantum mixing, Internat. Math. Res. Notices, 1996, pp. 115-137.

[Ze2] S. Zelditch, Szegö kernels and a theorem of Tian, Internat. Math. Res. Notices, 1998, pp. 317-331. 